Ido A. Iurgel Nelson Zagalo Paolo Petta (Eds.)

\title{
Interactive Storytelling
}

Second Joint International Conference on Interactive Digital Storytelling, ICIDS 2009 Guimarães, Portugal, December 9-11, 2009 Proceedings 


\section{Volume Editors}

Ido A. Iurgel

Universidade do Minho

DSI - Departamento de Sistemas de Informação

Campus de Azurém, 4800-058 Guimarães, Portugal

E-mail: ido.iurgel@dsi.uminho.pt

Nelson Zagalo

Universidade do Minho

Departamento de Ciências da Comunicação

Campus de Gualtar, 4710-057 Braga, Portugal

E-mail: nzagalo@ics.uminho.pt

Paolo Petta

Austrian Research Institute for Artificial Intelligence

Freyung 6/6, A 1010 Vienna, Austria

E-mail:paolo.petta@ofai.at

Library of Congress Control Number: 2009939506

CR Subject Classification (1998): J.5, H.3.1, I.2.1, K.8, K.3, J.4, I.7

LNCS Sublibrary: SL 3 - Information Systems and Application, incl. Internet/Web and $\mathrm{HCI}$

ISSN 0302-9743

ISBN-10 3-642-10642-0 Springer Berlin Heidelberg New York

ISBN-13 978-3-642-10642-2 Springer Berlin Heidelberg New York

This work is subject to copyright. All rights are reserved, whether the whole or part of the material is concerned, specifically the rights of translation, reprinting, re-use of illustrations, recitation, broadcasting, reproduction on microfilms or in any other way, and storage in data banks. Duplication of this publication or parts thereof is permitted only under the provisions of the German Copyright Law of September 9, 1965, in its current version, and permission for use must always be obtained from Springer. Violations are liable to prosecution under the German Copyright Law.

springer.com

(C) Springer-Verlag Berlin Heidelberg 2009

Printed in Germany

Typesetting: Camera-ready by author, data conversion by Scientific Publishing Services, Chennai, India Printed on acid-free paper $\quad$ SPIN: $12804450 \quad 06 / 3180 \quad 543210$ 


\section{Preface}

The rich programme of ICIDS 2009, comprising invited talks, technical presentations and posters, demonstrations, and co-located post-conference workshops clearly underscores the event's status as premier international meeting in the domain. It thereby confirms the decision taken by the Constituting Committee of the conference series to take the step forward: out of the national cocoons of its precursors, ICVS and TIDSE, and towards an itinerant platform reflecting its global constituency. This move reflects the desire and the will to take on the challenge to stay on the lookout, critically reflect upon and integrate views and ideas, findings and experiences, and to promote interdisciplinary exchange, while ensuring overall coherence and maintaining a sense of direction.

This is a significant enterprise: The challenges sought are multifarious and must be addressed consistently at all levels. The desire to involve all research communities and stakeholders must be matched by acknowledging the differences in established practises and by providing suitable means of guidance and introduction, exposition and direct interaction at the event itself and of lasting (and increasingly: living) documentation, of which the present proceedings are but an important part. To illustrate: for the scientific constituency, this implies solving the issue of how to achieve high impact (factors) for different disciplines with differing understandings of the role of conferences and conference publications; for artists, to accommodate their presentations to technology-oriented expectations; for the editors, this requires preparedness and availability to put up with the broader range of quirks typical of the different authoring communities, and to invest work on adjusting form (compliance with specific layouts) and ensuring quality of content (e.g., fighting the particular disease of all too frequent issues with correctness and completeness of documentation of the embedding of the original work presented that still marks the original manuscripts of the more recent scientific and engineering disciplines).

At first sight, all of this could be misinterpreted as adding up to a disproportionate amount of trouble. But in fact these are the tangible and lively signs of an example of how to do away with balkanisations and divisions into two (or however many) cultures.

The recognition of the role of ICIDS is reflected in the number of submissions received: almost double the number over the already very successful first edition, and with some spread across areas (if still with room for future improvement). Clearly, this did not make the work of the Programme Committee any easier, and we want to thank each of the members, as well as the auxiliary reviewers, for their diligent work: this includes the noteworthy additional effort invested in discussions and further rounds of inspection of contributions that could be accepted only conditionally at first, and in support of an open mindset. The final decisions of acceptance and of the format assigned to each contribution 
were thus based on close examination of all reviews and commentary received (as well as the inevitable influence of the logistic givens).

Out of 89 paper and poster submissions, the final programme comprised 19 full and 11 short paper presentations. Together with five accepted posters, this adds up to an overall acceptance rate of $39 \%$ that reflects well the aspired balance between the aims for inclusiveness and quality. Six demonstrations and four invited presentations provided further complementary samples of the interactive digital storytelling domain. In addition, a number of post-conference workshops offered the opportunity for more in-depth discussions, informed also by handson sessions, about selected topics. Out of the 108 co-authors of contributions to the main conference programme, 58 come from Europe, 31 from North America, ten from the Far East, four each from the Near East (Israel) and South America (Brazil), and one from Australia - with a noteworthy number of cross-continental collaborations.

In closing, we also wish to thank: all of the members of the ICIDS community who submitted; all the supporters of the ICIDS 2009 project and the sponsoring organisations; and in particular Uli Spierling and Nicolas Szilas, the organisers of the first ICIDS, for making our life easier in so many ways by not just handing over the torch, but providing continuous helpful commentary and suggestions; and, last but not least, the Constituting Committee for entrusting us with this important task.

October 2009

Paolo Petta

Nelson Zagalo

Ido A. Iurgel 